\title{
脱氧野尻霉素类两亲化合物的合成及其超分子糖苷酶抑制活性研究
}

\author{
李 敏 ${ }^{a, b}$ 刘茂华 ${ }^{a}$ 王 琪 ${ }^{a}$ 王克让 ${ }^{*}, a$ 李小六*,a \\ ( ${ }^{a}$ 河北大学化学与环境科学学院 药物化学与分子诊断教育部重点实验室 \\ 河北省化学生物学重点实验室 河北保定 071002) \\ $\left({ }^{b}\right.$ 邢台学院化学与化工学院 河北邢台 054001)
}

\begin{abstract}
摘要 以葡萄糖为原料经多步反应合成了脱氧野尻霉素类两亲化合物 FA-DNJ-C6, 通过表面张力实验、动态光散射实 验(DLS)和透射电镜(TEM)等研究了 FA-DNJ-C6 的自组装行为, FA-DNJ-C6 在水溶液中形成稳定的超分子自组装体. 经酶抑制实验研究了 FA-DNJ-C6 自组装体的糖苷酶抑制活性，进而发现 FA-DNJ-C6 自组装体对 $\alpha$-糖苷酶具有好的选 择性, 尤其是对 $\alpha$-甘露糖苷酶, 其抑制活性的 $K_{\mathrm{i}}$ 值为 $(0.107 \pm 0.021) \mu \mathrm{mol} / \mathrm{L}$, 与阳性对照(米格列醇)相比, 活性提高了 339 倍, 这主要是由于 $\alpha$-甘露糖苷酶具有多个识别位点的空腔, 可发挥多效价协同增强的键合作用, 提高糖苷酶抑制活 性.
\end{abstract}

关键词 糖苷酶抑制剂; 自组装; 多效价; 1-脱氧野尻霉素

\section{Synthesis and Glycosidase Inhibition Activity of an Amphiphilic Fatty-Deoxynojirimycin Derivative}
$\mathrm{Li}, \mathrm{Min}^{a, b}$
Liu, Maohua ${ }^{a}$
Wang, $\mathrm{Qi}^{a}$
Wang, Kerang ${ }^{*, a}$
Li, Xiaoliu ${ }^{*, a}$

( ${ }^{a}$ Key Laboratory of Medicinal Chemistry and Molecular Diagnosis (Ministry of Education), Key Laboratory of Chemical Biology of Hebei Province, College of Chemistry and Environmental Science, Hebei University, Baoding, Hebei 071002) $\left({ }^{b}\right.$ College of Chemistry and Chemical Engineering, Xingtai University, Xingtai, Hebei 054001)

\begin{abstract}
An amphiphilic derivative FA-DNJ-C6 with deoxynojirimycin modification was synthesized. The self-assembly behavior of FA-DNJ-C6 was studied by a surface tension test, dynamic light scattering test (DLS) and transmission electron microscopy (TEM). FA-DNJ-C6 formed stable supramolecular self-assembly in aqueous solution. Furthermore, the glycosidase inhibition activities of FA-DNJ-C6 were studied. FA-DNJ-C6 as multivalent glycosidase inhibitor showed potent glycosidase effect against $\alpha$-mannosidase with $K_{\mathrm{i}}$ value of $(0.107 \pm 0.021) \mu \mathrm{mol} / \mathrm{L}$, an increase of approximately 339 -fold compared with the control drug of miglitol, which was due to the multivalent binding sites in $\alpha$-mannosidase.
\end{abstract}

Keywords glycosidase inhibitor; self-assembly; multivalent; 1-deoxynojirimycin

糖苷酶对寡糖糖苷键具有专一性水解作用 ${ }^{[1]}$, 糖苷 酶的异常与糖尿病 ${ }^{[2]}$ 、戈谢病 ${ }^{[3]}$ 、癌症 ${ }^{[4]}$ 和病毒感染 ${ }^{[5]}$ 等疾病密切相关. 目前, 糖苷酶抑制剂被广泛用于降血. 糖药物和抗病毒药物 ${ }^{[6]}$. 近年来, 人们研究发现糖苷酶 抑制剂与糖苷酶之间的相互作用具有 “多价效应”或 “簇 糖效应” [7], 因此, 多效价糖苷酶抑制剂的研究引起人们 广泛关注 ${ }^{[7]}$, 其对糖苷酶具有更高的选择性和更好的抑 制活性 ${ }^{[8]}$. 如 14 个 1-脱氧野尻霉素(DNJ)分子修饰的环 糊精簇糖分子对 $\alpha$-甘露糖苷酶的抑制活性的 $K_{\mathrm{i}}$ 值为 22 $\mathrm{nmol} / \mathrm{L}$ ，折合单效价活性比单体化合物提高了 610 倍 ${ }^{[9]}$. 120 个和 108 个 1 -脱氧野尻霉素分子修饰的富勒烯 化合物对 $\alpha$-甘露糖苷酶的抑制活性 $\left(K_{\mathrm{i}}\right)$ 分别为 1.8 和 7.2 $\mathrm{nmol} / \mathrm{L}^{[10]}$ ，折合单效价活性比单体化合物活性分别高 944 倍和 262 倍. 尽管多效价糖苷酶抑制剂的研究取得 了较好的成果，但共价键多步合成的笶糖分子存在合成 路线长和分离纯化困难等问题.

超分子自组装是构筑多效价簇糖分子的另一有效 途径 ${ }^{[11]}$, 具有合成步骤相对简单、分离纯化容易和组装

\footnotetext{
* Corresponding authors. E-mail: kerangwang@hbu.edu.cn; lixl@hbu.edu.cn

Received August 14, 2019; revised September 24, 2019; published online October 25, 2019.

Project supported by the National Natural Science Foundation of China (Nos. 21572044, 21778013), the Natural Science Foundation of Hebei Province (No. B2016201254), and the Foundation of Hebei Education Department (No. QN2018310).

国家自然科学基金(Nos. 21572044, 21778013)、河北省自然科学基金(No. B2016201254)和河北省教育厅研究(No. QN2018310)资助项目.
} 
过程可调控等优点 ${ }^{[12]}$. 迄今为止, 自组装多效价糖基自 组装体已被用于抑制流感病毒感染 ${ }^{[13]}$ 、凝集素识别 ${ }^{[14]}$ 和蛋白分子的捕获和释放 ${ }^{[15]}$ 等方面的研究. 然而, 自组 装多效价糖苷酶抑制剂的研究较少. Compain 和 Lecommandoux 等 ${ }^{[16]}$ 报道了基于多肽-野尻霉素分子的自组 装多效价糖苷酶抑制剂, 其对 $\alpha$-甘露糖苷酶的抑制活性 $\left(K_{\mathrm{i}}\right)$ 为 $0.15 \mu \mathrm{mol} / \mathrm{L}$, 比单体化合物的活性提高了 206 倍, 该研究结果说明自组装方法是构筑多效价糖苷酶抑制 剂的有效途径. 最近, 我们课题组 ${ }^{[17]}$ 制备了 6 个 1-脱氧 野尻需素分子修饰的菲酰亚胺化合物 PBI-DNJ, 其自组 装形成的超分子多效价糖苷酶抑制剂对 $\alpha$-糖苷酶具有 好的选择性, 尤其是对 $\alpha$-甘露糖苷酶, 其抑制活性 $\left(K_{\mathrm{i}}\right)$ 为 $38 \mathrm{nmol} / \mathrm{L}$, 与米格列醇 (上市药物)相比活性提高了 460 倍. 为了改善自组装多效价糖苷酶抑制剂的生物相 容性, 制备了 1-脱氧野尻霉修饰的脂肪胺衍生物 FADNJ, 其中长链柔性脂肪胺基团具有自动调节识别位点 的特点 ${ }^{[18]}$. 基于 FA-DNJ 的自组装多效价糖苷酶抑制剂 对 $\alpha$-甘露糖苷酶的抑制活性 $\left(K_{\mathrm{i}}\right)$ 为 $0.11 \mu \mathrm{mol} / \mathrm{L}$, 与米格 列醇(上市药物)相比活性提高了 330 倍 ${ }^{[18]}$. 根据文献报 道, 1-脱氧野尻霉素基团连接链的长度直接影响着化合 物的糖苷酶抑制活性 ${ }^{[8 c, 9,19]}$, 如己基连接的 1-脱氧野尻 霉素衍生物的活性好于丙基连接链的化合物, 而壬基连 接的化合物好于己基连接的化合物 ${ }^{[8 c, 9,19]}$.

为了研究连接链长度对 1-脱氧野尻霉素衍生物活 性的影响, 并兼顾该化合物的水溶性以利于后续活性研 究, 合成了己基连接的 1-脱氧野尻霉素修饰的脂肪胺分 子 FA-DNJ-C6 (图 1), 通过表面张力实验、动态光散射 实验(DLS)和透射电镜(TEM)等研究了 FA-DNJ-C6 的自 组装行为, FA-DNJ-C6 在水溶液中形成稳定的超分子自 组装体. 进而, 经酶抑制实验研究了 FA-DNJ-C6 自组 装体的糖苷酶抑制活性, 发现 FA-DNJ-C6 自组装体

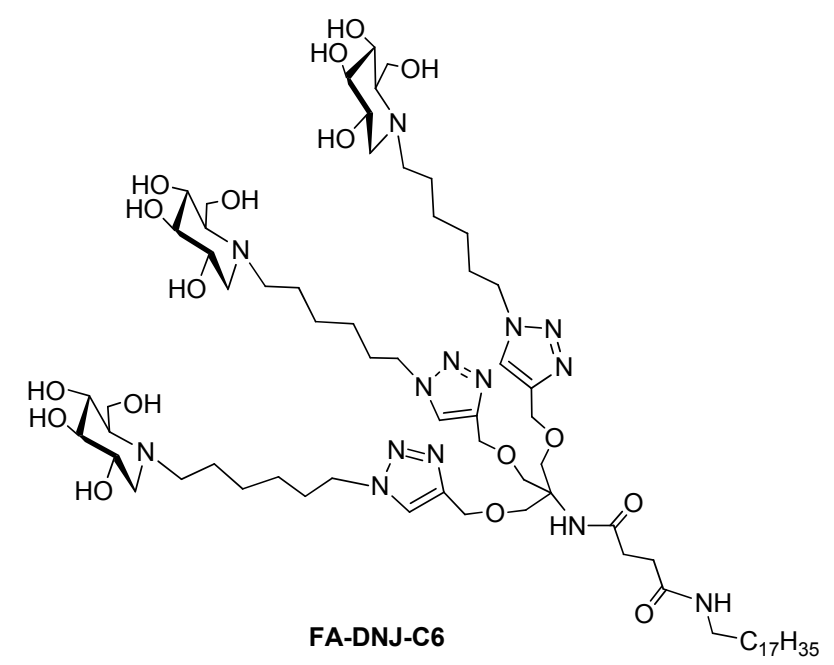

图 1 FA-DNJ-C6 的结构

Figure 1 Structure of compound FA-DNJ-C6
对 $\alpha$-糖苷酶具有好的选择性，尤其是对 $\alpha$-甘露糖苷酶, 其抑制活性的 $K_{i}$ 值为 $0.107 \mu \mathrm{mol} / \mathrm{L}$ ，与阳性对照(米格列 醇)相比，活性提高了 339 倍.

\section{1 结果与讨论}

\subsection{FA-DNJ-C6 化合物的合成}

如 Scheme 1 所示, 以葡萄糖为原料, 在三氟化嗍乙 醚催化下与甲醇反应生成 1-甲基葡萄糖糖苷(1), 其它 差基在碱性条件下用苄基保护制备了中间体 2.2 在乙 酸/硫酸 $(V: V=3: 1)$ 混合溶液中选择性脱除 1-位甲基 制备了 3. 进而 3 在 $\mathrm{LiAlH}_{4}$ 作用下还原生成二羟基衍生 物 4. 在一 $78{ }^{\circ} \mathrm{C}$ 下用二甲基亚砜(DMSO)与草酰氯共同 作用，将 4 氧化成二醛中间体，再经 Mannich 反应，关环 生成亚胺化合物 $\mathbf{5}^{[20]}$. 在氢氧化钯/碳催化下, 5 加氢还 原脱去苄基生成 6.6 与溴已基叠氮化合物发生亲核取代 反应生成叠氮己基脱氧野尻霉素衍生物, 为了分离纯化 需要, 进一步与乙酸酐反应生成乙酰基保护的 1-脱氧野 尻毒素衍生物 7. 最后通过 Click 反应与我们文献中报道 的炔基中间体 $\mathbf{8}^{[18]}$ 反应生成 FA-AcDNJ-C6, 脱除乙酰 基制备了目标化合物 FA-DNJ-C6. FA-DNJ-C6 及其中 间体经 NMR 和 HRMS 等表征.

\section{2 自组装行为研究}

通过悬滴法研究了 FA-DNJ-C6 在水溶液中的自组 装行为. 配制 FA-DNJ-C6 不同浓度的溶液 $\left(4 \times 10^{-6} \sim\right.$ $\left.5 \times 10^{-4} \mathrm{~mol} / \mathrm{L}\right)$ ，经接触角测量仪测定不同浓度下的表 面张力 $(\mathrm{ST})^{[21]}$. 如图 2 所示, FA-DNJ-C6 在浓度低于 $1 \times 10^{-5} \mathrm{~mol} / \mathrm{L}$ 时, 表面张力与水的表面张力类似. 随着 FA-DNJ-C6 浓度的增加，溶液表面张力迅速降低，当浓 度大于 $3 \times 10^{-4} \mathrm{~mol} / \mathrm{L}$ 时, 溶液 $\gamma$ 值不再变化. 在拟合曲 线拐点处作切线, 两条切线交于一点, 此点对应的浓度 为最小聚集浓度 $\left(\mathrm{CAC}, 9.20 \times 10^{-5} \mathrm{~mol} / \mathrm{L}\right)$, 比我们报道 的丙基衍生物 FA-DNJ 略高 $\left(6.32 \times 10^{-5} \mathrm{~mol} / \mathrm{L}\right)$, 表面张 力为 $47.39 \mathrm{mmol} \cdot \mathrm{L}^{-1} \cdot \mathrm{m}^{-1}$, 比 FA-DNJ $(50.3 \mathrm{mmol}$. $\mathrm{L}^{-1} \cdot \mathrm{m}^{-1}$ )略低, 这主要是由于连接亲水基团的支链增长, 增加了化合物的 CAC 值而降低了其表面张力 ${ }^{[22]}$. 这些 研究结果表明 FA-DNJ-C6 具有较高的表面活性, 在水 溶液中易形成自组装体 ${ }^{[23]}$.

进而, 通过动态光散射(DLS)实验研究了 FA-DNJC6 在不同 $\mathrm{pH}$ 值下自组装体的稳定性. 在酸性条件下 $(\mathrm{pH}=2.0 \sim 4.0), \mathbf{F A - D N J}-\mathbf{C 6}$ 自组装体的平均粒径分别 为 159, 164 和 $157 \mathrm{~nm}$ (图 3), 说明 FA-DNJ-C6 在酸性条 件下能形成稳定的自组装体. 而在 $\mathrm{pH}$ 值为 5.0, 6.0 和 7.0 时, FA-DNJ-C6 自组装体的平均粒径分别为 130,128 和 $131 \mathrm{~nm}$ (图 3). 实验结果表明 FA-DNJ-C6 在酸性条件 下形成较大的自组装体, 这主要是由于酸性条件下 1-脱 

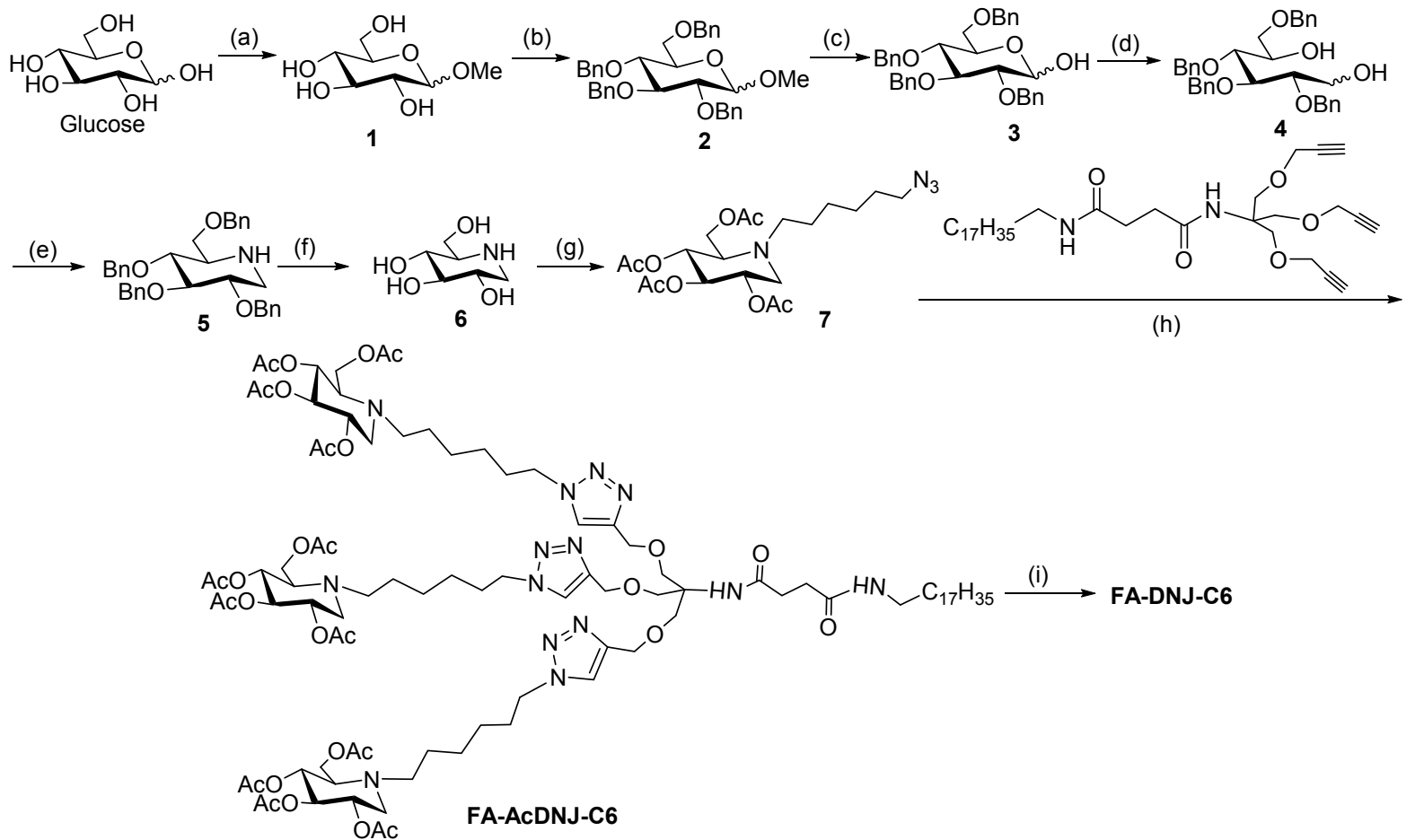

Reagents and conditions: (a) $\mathrm{BF}_{3} \cdot \mathrm{Et}_{2} \mathrm{O}, \mathrm{MeOH}$, reflux, $10 \mathrm{~h}, 77.9 \%$; (b) $\mathrm{NaH}, \mathrm{BnCl}$, DMF, r.t., $20 \mathrm{~h}, 78.4 \%$; (c) $\mathrm{AcOH} / \mathrm{H}_{2} \mathrm{SO}_{4}(\mathrm{~V} / \mathrm{V}=3 / 1), 80{ }^{\circ} \mathrm{C}, 5 \mathrm{~h}$, 82.1\%; (d) $\mathrm{LiAlH}_{4}$, THF, r.t., $20 \mathrm{~h}, 85 \%$; (e) $\mathrm{Et} 3 \mathrm{~N}$, DMSO, $(\mathrm{COCl})_{2}$, DCM, $-78{ }^{\circ} \mathrm{C}, 6$ h; $\mathrm{NaCNBH}_{3}, \mathrm{NH}_{4} \mathrm{HCO}_{3}, \mathrm{Na}_{2} \mathrm{SO}_{4}, \mathrm{MeOH}$, r.t., 12 h, $62 \%$; (f) $\mathrm{H}_{2}$, $\mathrm{Pd}(\mathrm{OH})_{2} / \mathrm{C}, \mathrm{MeOH}, 1 \mathrm{~mol} / \mathrm{L} \mathrm{HCl}$, r.t., $10 \mathrm{~h}, 96 \%$; (g) $\mathrm{K}_{2} \mathrm{CO}_{3}$, DMF, 6-azidohexyl bromide, $110{ }^{\circ} \mathrm{C}, 24 \mathrm{~h}$; $\mathrm{Ac}_{2} \mathrm{O}$, $\mathrm{Py}$, r.t., 10 h, $50 \%$; (h) $\mathrm{CuSO}_{4} \cdot 5 \mathrm{H}_{2} \mathrm{O}$, L-sodium ascorbate, THF- $\mathrm{H}_{2} \mathrm{O}, 55{ }^{\circ} \mathrm{C}, 10 \mathrm{~h}, 64 \%$; (i) $\mathrm{NaOMe}, \mathrm{MeOH}, 92 \%$

图式 1 FA-DNJ-C6 的合成

Scheme 1 Synthesis of FA-DNJ-C6

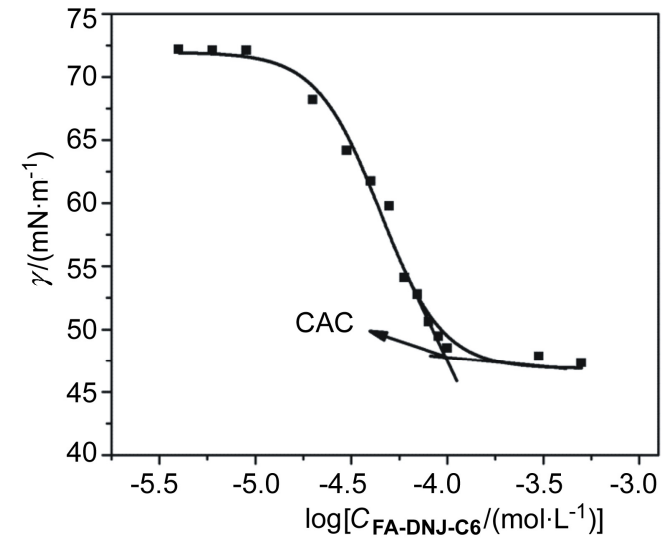

图 $225{ }^{\circ} \mathrm{C}$ 时不同浓度下 FA-DNJ-C6 的表面张力 $(\gamma)$ 与 $\log$ $\left[C_{\mathrm{FA}-\mathrm{DNJ}-\mathrm{C6} 6} /\left(\mathrm{mol} \cdot \mathrm{L}^{-1}\right)\right]$ 曲线图 $(\mathrm{pH}=7.0)$

Figure 2 Plot and fitting curve of ST $(\gamma)$ versus $\log \left[C_{\text {FA-DNJ-C6 }}\right]$ $\left.\left(\mathrm{mol} \cdot \mathrm{L}^{-1}\right)\right]$ in PBS buffer $(\mathrm{pH}=7.0)$ at $25{ }^{\circ} \mathrm{C}$

氧野尻霉素分子中 $\mathrm{N}$ 原子质子化使分子间斥力增加, 导 致自组装体粒径增大 ${ }^{[24]}$. 与我们报道的丙基衍生物 FA-DNJ 相比, FA-DNJ-C6 由于增长的碳链导致自组装 体尺寸增大.

透射电子显微镜(TEM) 可直观地观察到自组装体的 组装形貌特征, 在 $1.0 \times 10^{-4} \mathrm{~mol} / \mathrm{L}$ 浓度下, FA-DNJ-C6

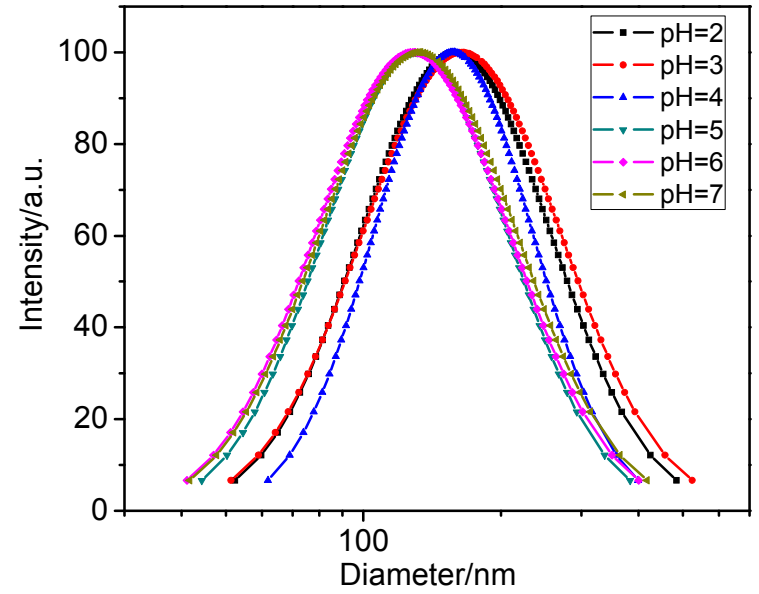

图 3 FA-DNJ-C6 $\left(1.0 \times 10^{-4} \mathrm{~mol} / \mathrm{L}\right)$ 在不同 $\mathrm{pH}(2.0 \sim 7.0)$ 值的 柠檬酸-磷酸盐缓冲液中的粒径分布

Figure 3 DLS results of the self-assemblies of FA-DNJ-C6 at different $\mathrm{pH}$ values $(\mathrm{pH} 2.0 \sim 7.0)$ in citric acid-phosphate buffer at the concentration of $1.0 \times 10^{-4} \mathrm{~mol} / \mathrm{L}$

的 TEM 结果显示其自组装形成球形自组装体, 组装体 直径在 80 和 $140 \mathrm{~nm}$ 之间(图 4), 与 DLS 结果一致.

\section{3 糖苷酶抑制活性研究}

FA-DNJ-C6 在水溶液中形成自组装体, 可能具有 自组装多效价糖苷酶抑制活性. 研究了 FA-DNJ-C6 对 


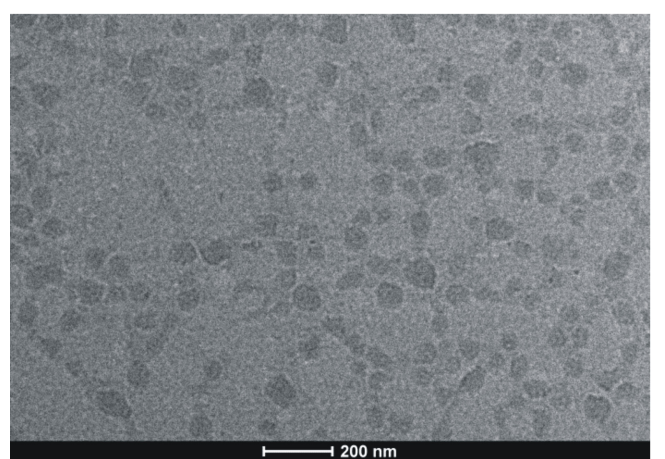

图 4 FA-DNJ-C6 的 TEM 图

Figure 4 TEM image of the self-assembly of FA-DNJ-C6

$\alpha$-甘露糖苷酶(Jack bean)、 $\beta$-甘露糖苷酶(Helix pomatia)、 $\alpha$-半乳糖苷酶(Green coffee bean)、 $\beta$-半乳糖苷 酶(Escherichia coli)、 $\alpha$-葡萄糖苷酶(Aspergillus niger)和 $\beta$-葡萄糖苷酶(almonds)等不同糖苷酶的体外抑制活性.

根据文献 [8c]方法, 测定了对硝基苯酚 (PNP) 和邻 硝基苯酚 $(\mathrm{ONP})$ 在柠檬酸-磷酸盐缓冲液 $(\mathrm{pH}=5.5,0.1$ $\mathrm{mmol} / \mathrm{L}$ ) 和 $\mathrm{PBS}$ 缓冲液 $(\mathrm{pH}=6.8$ 或 $7.3,0.1 \mathrm{mmol} / \mathrm{L})$ 中的 标准曲线. 分别以米格列醇(上市药物)和单体化合物 DNJ-1 为阳性对照, 研究了 FA-DNJ-C6 的糖苷酶抑制
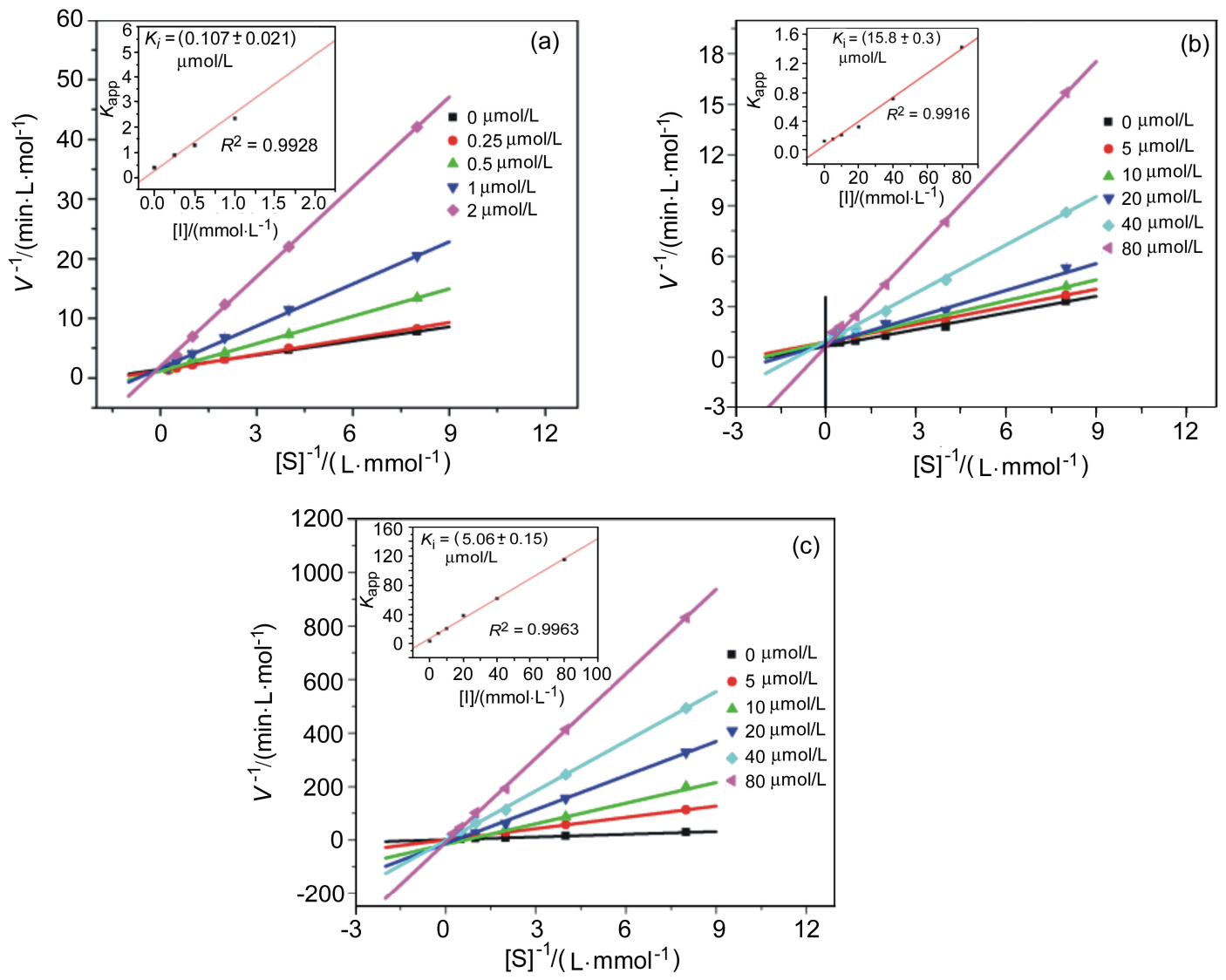

图 5 FA-DNJ-C 抑制 $\alpha$-甘露糖苷酶(a)、 $\alpha$-半乳糖苷酶(b)和 $\alpha$-葡萄糖苷酶(c)的 Line weaver-Burk 曲线

Figure 5 Lineweaver-Burk plot for $K_{\mathrm{i}}$ determination of FA-DNJ-C6 against $\alpha$-mannosidase (a), $\alpha$-galactosidase (b) and $\alpha$-glucosidase (c) 
表 1 FA-DNJ-C6 的糖苷酶抑制活性 $\left(K_{\mathrm{i}}, \mu \mathrm{mol} \cdot \mathrm{L}^{-1}\right)$ 、相对效价 $(\mathrm{rp})$ 和相对单效价 $(\mathrm{rp} / n)$

Table 1 Glycosidase inhibitory activities $\left(K_{\mathrm{i}}, \mu \mathrm{mol} \cdot \mathrm{L}^{-1}\right)$ of FA-DNJ-C6, relative inhibition potency (rp) over miglitol/FA-DNJ-1 and relative inhibition potency $(\mathrm{rp} / n)$ over rp/per DNJ unit

\begin{tabular}{lllllll}
\hline 糖苷酶 & $\alpha$-甘露糖苷酶 & $\beta$-甘露糖苷酶 & $\alpha$-半乳糖苷酶 & $\beta$-半乳糖苷酶 & $\alpha$-葡萄糖苷酶 & $\beta$-葡萄糖苷酶 \\
\hline FA-DNJ-C6 & $0.107 \pm 0.021$ & $\mathrm{NI}^{a}$ & $15.8 \pm 0.3$ & $\mathrm{NI}^{a}$ & $5.06 \pm 0.15$ & $\mathrm{NI}^{a}$ \\
Miglitol & $109 \pm 3^{[18]}$ & $\mathrm{NI}^{a}$ & $\mathrm{NI}^{a}$ & $\mathrm{NI}^{a}$ & $20.64 \pm 0.04^{[17]}$ & $\mathrm{NI}^{a}$ \\
$\mathbf{D N J - 1}$ & $445 \pm 12$ & $\mathrm{NI}^{a}$ & $71.3 \pm 0.1$ & $\mathrm{NI}^{a}$ & $1.60 \pm 0.3$ & $\mathrm{NI}^{a}$ \\
$\mathbf{D N J}^{[25]}$ & 270 & $\mathrm{NI}^{a}$ & $\mathrm{NI}^{a}$ & 42 & 25 & 47 \\
$\mathrm{rp}$ & $1018 / 4158$ & & & & 4.07 & \\
$\mathrm{rp} / n^{c}$ & $339 / 1386$ & & & & 1.36 & \\
\hline
\end{tabular}

${ }^{a} \mathrm{NI}$ : no inhibition detected at $1 \mathrm{mmol} / \mathrm{L} .{ }^{b}$ Relative inhibition potency (rp) over miglitol/FA-DNJ-1 ${ }^{c}$ Relative inhibition potency (rp/n) over rp/per DNJ unit.

多效价 1-脱氧野尻霉素类衍生物对 $\alpha$-甘露糖苷酶具有 更好的活性.

\section{2 结论}

合成了一种 1-脱氧野尻霉素修饰脂肪基衍生物 FA-DNJ-C6, 通过表面张力、动态光散射和 TEM 实验 等研究了其自组装行为, 发现化合物 FA-DNJ-C6 自组 装形成了 $80 \sim 140 \mathrm{~nm}$ 球形组装体, 并且该自组装体在 $\mathrm{pH}$ 值为 $2.0 \sim 7.0$ 溶液中能稳定存在. 进而, 通过体外糖 苷酶抑制实验研究了化合物 FA-DNJ-C6 自组装体对不 同糖苷酶的抑制活性，发现其对 $\alpha$-糖苗酶具有好的选择 性, 尤其是对 $\alpha$-甘露糖苷酶的抑制活性的 $K_{\mathrm{i}}$ 值为 $(0.107 \pm 0.021) \mu \mathrm{mol} / \mathrm{L}$, 比米格列醇(Miglitol)的活性高 出 1018 倍, 比单价化合物 DNJ-1 的活性高出 4158 倍, 相对单效价分别提高了 339 和 1386 倍. 碳链增长导致 FA-DNJ-C6 自组装体的粒径与丙基连接链相比增大了 $20 \mathrm{~nm}$, 糖苷酶抑制活性都有所提高, 但是对 $\alpha$-甘露糖 苷酶的抑制活性变化不大. 研究结果表明, 改变连接 $\mathrm{DNJ}$ 分子碳链长度, 脂肪基两亲 1-脱氧野尻霉素衍生物 的组装方式变化不大, 但可以增加对糖苷酶的抑制活 性. 多效价 1-脱氧野尻霉素类衍生物对 $\alpha$-甘露糖苷酶具 有更好的活性的原因是 $\alpha$-甘露糖苷酶具有多个识别位 点的空腔, 可发挥多效价协同增强的键合作用, 提高糖 苷酶抑制活性.

\section{3 实验部分}

\section{1 仪器与试剂}

${ }^{1} \mathrm{H}$ NMR 和 ${ }^{13} \mathrm{C}$ NMR 用 Bruker AVANCE $600 \mathrm{MHz}$ (TMS 为内标)核磁共振波谱仪测量; 高分辨率质谱用布 鲁克 FTICR-MD 质谱仪测定; 表面张力用接触角测量仪 OCA 15EC 测定; 粒径用动静态光散射仪美国 Brookhaven BI-APDV 测定; TEM 用透射电镜 Tecnai G2 F20 S-TWIN 测定; 酶抑制活性用酶标仪 BioTek 测定.

\section{2 化合物的制备}

\subsection{1葡萄糖衍生物 $\mathbf{1}$ 的合成}

$250 \mathrm{~mL}$ 的圆底烧瓶中加入葡萄糖 $(10.0 \mathrm{~g}, 55.6$ $\mathrm{mmol}$ )和 $100 \mathrm{~mL}$ 无水甲醇, 加入催化量的三氟化硼乙 醚, 氮气保护下加热回流 $12 \mathrm{~h}$, 加入三乙胺淬灭反应, 并调节 $\mathrm{pH}$ 值至中性，旋干溶剂，经硅胶(200 300 目) 柱色谱分离 $[V$ (乙酸乙酯) $/ V$ (甲醇 $)=8 / 1$ 洗脱], 得到白色 固体 1, $8.4 \mathrm{~g}$, 产率 $77.9 \%$, m.p. $160.5 \sim 162{ }^{\circ} \mathrm{C}$. 谱图数 据与文献[20]一致.

\section{2 .2 葡萄糖衍生物 $\mathbf{2}$ 的合成}

$250 \mathrm{~mL}$ 的圆底烧瓶中加入 $\mathrm{NaH}(4.9 \mathrm{~g}, 0.20 \mathrm{~mol})$ 和 无水 DMF $(50 \mathrm{~mL})$ ，搅拌下加入葡萄糖衍生物 $1(5.0 \mathrm{~g}$, $25.7 \mathrm{mmol})$, 滴加 $\mathrm{BnCl}(19.5 \mathrm{~g}, 0.15 \mathrm{~mol})$, 室温搅拌 10 $\mathrm{h}$, 滴加甲醇至无气体生成, 再加入大量二氯甲烷, 水洗 除去 DMF. 有机相用无水硫酸钠干燥, 旋干溶剂, 经柱 色谱分离 $[V$ (石油醚 $) / V($ 乙酸乙酯 $)=8 / 1$ 洗脱 $]$, 得到淡黄 色浆状物质 2, $11.2 \mathrm{~g}$ ，产率 78.4\%. 谱图数据与文献[20] 一致.

\subsection{3 葡萄糖衍生物 $\mathbf{3}$ 的合成}

$250 \mathrm{~mL}$ 的圆底烧瓶中加入葡萄糖衍生物 $2(3.0 \mathrm{~g}$, $5.4 \mathrm{mmol}$ ), 将 $100 \mathrm{~mL}$ 冰醋酸与 $3 \mathrm{~mol} / \mathrm{L}$ 硫酸(体积比为 $3 / 1$ 的混合溶液加入烧瓶中, 氮气保护下置于 $80{ }^{\circ} \mathrm{C}$ 油 浴中加热搅拌反应，薄层色谱(TLC)监测反应. 反应结 束后静置冷却至室温, 析出白色针状晶体, 抽滤, 固体 用水洗至滤液呈中性，真空干燥得到白色固体 $3,2.4 \mathrm{~g}$, 产率 $82.1 \%$, m.p. $51.4 \sim 53.2{ }^{\circ} \mathrm{C}$. 谱图数据与文献[20]一 致.

\subsection{4 葡萄糖衍生物 $\mathbf{4}$ 的合成}

在 $250 \mathrm{~mL}$ 的圆底烧瓶中加入葡萄糖衍生物 $3(2.0$ $\mathrm{g}, 3.70 \mathrm{mmol})$ 与 $100 \mathrm{~mL}$ 无水 $\mathrm{THF}$, 冰水浴下加入氢化铝 锂 $(280 \mathrm{mg}, 7.40 \mathrm{mmol})$, 氮气保护下室温摚拌反应 $4 \mathrm{~h}$, TLC 监测反应. 冰水浴下加水水解过量的氢化铝锂, 加 入 $200 \mathrm{~mL}$ 乙酸乙酯稀释, 用 $50 \mathrm{~mL}$ 饱和氯化铵溶液洗 
三次，有机相用无水硫酸钠干燥，旋干溶剂得淡黄色粘 稠液体, 经硅胶 $(200 \sim 300$ 目) 柱色谱分离 $[V$ (石油 醚 $) / V($ 乙酸乙酯 $)=3 / 1$ ] 得到淡黄色浆状物质 $4,1.7 \mathrm{~g}$, 产 率 $85 \%$. 谱图数据与文献 $[20]$ 一致.

3.2.5 葡萄糖衍生物 $\mathbf{5}$ 的合成

在 $250 \mathrm{~mL}$ 的圆底烧瓶中加入二氯甲烷(DCM) $(7.2$ $\mathrm{mL})$ 和草酰氯 $(1.272 \mathrm{~mL}, 14.7 \mathrm{mmol}),-78{ }^{\circ} \mathrm{C}$ 下向溶液 中滴加 DMSO (1.384 mL, $18.4 \mathrm{mmol}),-78{ }^{\circ} \mathrm{C}$ 下搅拌 $30 \mathrm{~min}$. 将葡萄糖衍生物 $4(2.0 \mathrm{~g}, 3.68 \mathrm{mmol})$ 溶于 DCM 并滴加入反应体系, $-78{ }^{\circ} \mathrm{C}$ 下搅拌 $2 \mathrm{~h}$, 再滴加三乙胺 (7.4 mL, $44.2 \mathrm{mmol}$ )的 DCM 溶液, 并慢慢升温至室温. $1000 \mathrm{~mL}$ 烧杯中加入 $200 \mathrm{~mL}$ 甲醇和 $600 \mathrm{~mL}$ 蒸馏水, 冰 水浴下加入碳酸氢铵 $(7.84 \mathrm{~g}, 99 \mathrm{mmol})$ 、氯基硼氢化钠 (0.982 g, $14.7 \mathrm{mmol})$ 和无水硫酸钠 $(2.226 \mathrm{~g}, 16.7 \mathrm{mmol})$. 将升至室温的反应液加入甲醇混合物中, 室温摚拌 20 h. DCM 萃取, 有机相用无水硫酸钠干燥, 浓缩得淡黄 色粘稠液体, 经硅胶 $(200 \sim 300$ 目) 柱色谱分离 $[V$ (石二 氯甲烷 $) / V$ (乙酸乙酯 $)=8 / 1$ ], 得到淡黄色固体 $5,1.2 \mathrm{~g}$, 产率 $62 \%$, m.p. $50.6 \sim 51.8{ }^{\circ} \mathrm{C}$. 谱图数据与文献[20]一 致.

\subsubsection{DNJ 行生物 6 的合成}

在氢气反应釜中加入 $\mathrm{MeOH}$ 和 $1 \mathrm{~mol} / \mathrm{L} \mathrm{HCl}$ 溶液 $(40$ $\mathrm{mL}, V / V=3 / 1)$ 溶解葡萄糖衍生物 $\mathbf{5}(1.0 \mathrm{~g}, 1.9 \mathrm{mmol})$, 再 加入 $\mathrm{Pd}(\mathrm{OH})_{2} / \mathrm{C}(300 \mathrm{mg})$, 在 $2.0 \mathrm{MPa}$ 氢气中催化反应 $24 \mathrm{~h}$, TLC 监测反应. 反应液经硅藻土抽滤得黄绿色溶 液, 旋蒸浓缩得淡黄色粘稠液体, 加入 $2 \mathrm{~mL}$ 甲醇, 超声 搅拌后析出白色晶体, 抽滤, 真空干燥得到白色固体 0.3 g. 产率 $96 \%$, m.p. $57.3 \sim 58.6{ }^{\circ} \mathrm{C}$. 谱图数据与文献 [20]一致.

\subsubsection{DNJ 行生物 7 的合成}

在 $100 \mathrm{~mL}$ 烧瓶中加入 DNJ 衍生物 $6(1 \mathrm{~g}, 6.1$ $\mathrm{mmol}$ ), 用 $5 \mathrm{~mL}$ 无水 DMF 溶解, 然后加入碳酸钾(1.69 $\mathrm{g}, 12.3 \mathrm{mmol}$ )及 6 -叠氮基己基溴 $(2.52 \mathrm{~g}, 12.3 \mathrm{mmol}$ ), $95{ }^{\circ} \mathrm{C}$ 加热反应 $12 \mathrm{~h}, \mathrm{TLC}$ [ $V$ (二氯甲烷 $) /[V$ (甲醇 $)=3 / 1$ ] 监测反应. 反应完毕, 减压浓缩除去 DMF, 加入 $10 \mathrm{~mL}$ 无水吡啶溶解, 在冰水浴下加入 DMAP, 滴加醋酸䣶 (3.75 mL, $36.8 \mathrm{mmol}$ ), 室温反应 $10 \mathrm{~h}$, TLC [ $V$ (石油 醚 $) / V($ 乙酸乙酯 $)=3 / 1]$ 监测反应. 反应完毕, 旋干吡啶, 硅胶 (200 300 目)柱色谱分离 $[V$ (石油醚) $/ V$ (乙酸乙 酯 $)=5 / 1$ ], 得到淡黄色粘稠液体 7, $1.4 \mathrm{~g}$, 产率 $50 \%$, m.p. $57.3 \sim 58.6{ }^{\circ} \mathrm{C} .{ }^{1} \mathrm{H}$ NMR $\left(400 \mathrm{MHz}, \mathrm{CDCl}_{3}\right) \delta: 5.03 \sim 5.11$ $(\mathrm{m}, 2 \mathrm{H}), 5.00 \sim 4.94(\mathrm{~m}, 1 \mathrm{H}), 4.22 \sim 4.10(\mathrm{~m}, 2 \mathrm{H}), 3.27(\mathrm{t}$, $J=6.8 \mathrm{~Hz}, 2 \mathrm{H}), 3.22 \sim 3.18(\mathrm{~m}, 1 \mathrm{H}), 2.77 \sim 2.71(\mathrm{~m}, 1 \mathrm{H})$, $2.63(\mathrm{~d}, J=8.4 \mathrm{~Hz}, 1 \mathrm{H}), 2.59 \sim 2.53(\mathrm{~m}, 1 \mathrm{H}), 2.35(\mathrm{t}, J=$ $10.8 \mathrm{~Hz}, 1 \mathrm{H}), 2.09$ (s, 3H), 2.03 (s, 6H), 2.02 (s, 3H), 1.62 (s, 4H), $1.43 \sim 1.35(\mathrm{~m}, 2 \mathrm{H}), 1.33 \sim 1.25(\mathrm{~m}, 2 \mathrm{H}) ;{ }^{13} \mathrm{C}$ NMR $\left(100 \mathrm{MHz}, \mathrm{CDCl}_{3}\right) \delta: 170.89,170.36,170.04$, $169.74,74.65,69.47,61.54,59.49,52.85,51.31,48.49$, $26.71,26.56,24.27,20.85,20.75,20.68,20.62$; HRMS (MALDI-TOF) calcd for $\mathrm{C}_{20} \mathrm{H}_{33} \mathrm{O}_{8} \mathrm{~N}_{4}$ 457.2286, found 457.2292.

\subsection{8 化合物 FA-AcDNJ-C6 的合成}

在 $50 \mathrm{~mL}$ 圆底烧瓶中加入化合物 8 (104 mg, 0.177 $\mathrm{mmol}) 、$ 化合物 7 (364 $\mathrm{mg}, 0.80 \mathrm{mmol})$ 和 THF $(15 \mathrm{~mL})$, $\mathrm{CuSO}_{4} \cdot 5 \mathrm{H}_{2} \mathrm{O}(79.7 \mathrm{mg}, 0.32 \mathrm{mmol})$ 和抗坏血酸钠 $(63 \mathrm{mg}$, $0.32 \mathrm{mmol}$ )水溶液, $55{ }^{\circ} \mathrm{C}$ 反应 $10 \mathrm{~h}$, 浓缩溶剂并经硅胶 $\left(200 \sim 300\right.$ 目)柱色谱分离 $\left[V\left(\mathrm{CH}_{2} \mathrm{Cl}_{2}\right) / V(\mathrm{MeOH})=20 / 1\right]$ 纯化, 得到淡黄色粘稠状物质 $222 \mathrm{mg}$, 产率 $64 \% .{ }^{1} \mathrm{H}$ NMR (600 MHz, $\mathrm{CDCl}_{3}$ ) $\delta: 7.58$ (s, 3H, Triazole-H), 6.41 (s, $1 \mathrm{H}, \mathrm{NH}), 6.26(\mathrm{~s}, 1 \mathrm{H}, \mathrm{NH}), 5.07 \sim 5.01(\mathrm{~m}, 6 \mathrm{H}), 4.96 \sim$ $4.93(\mathrm{~m}, 3 \mathrm{H}), 4.57(\mathrm{~s}, 6 \mathrm{H}), 4.35(\mathrm{t}, J=7.2 \mathrm{~Hz}, 6 \mathrm{H}), 4.15 \sim$ $4.13(\mathrm{~m}, 6 \mathrm{H}), 3.78(\mathrm{~s}, 6 \mathrm{H}), 3.19 \sim 3.14(\mathrm{~m}, 3 \mathrm{H}), 2.75 \sim$ $2.70(\mathrm{~m}, 3 \mathrm{H}), 2.62(\mathrm{~d}, J=9.0 \mathrm{~Hz}, 3 \mathrm{H}), 2.54 \sim 2.46(\mathrm{~m}$, $5 \mathrm{H}), 2.42 \sim 2.40(\mathrm{~m}, 2 \mathrm{H}), 2.30(\mathrm{t}, J=10.8 \mathrm{~Hz}, 3 \mathrm{H}), 2.06(\mathrm{~s}$, $\left.9 \mathrm{H}, \mathrm{CH}_{3}\right), 2.01\left(\mathrm{~s}, 18 \mathrm{H}, \mathrm{CH}_{3}\right), 2.00\left(\mathrm{~s}, 9 \mathrm{H}, \mathrm{CH}_{3}\right), 1.95 \sim$ $1.89(\mathrm{~s}, 6 \mathrm{H}), 1.45(\mathrm{~s}, 6 \mathrm{H}), 1.34(\mathrm{~s}, 10 \mathrm{H}), 1.23 \sim 1.26(\mathrm{~m}$, $34 \mathrm{H}), 0.88(\mathrm{t}, J=7.2 \mathrm{~Hz}, 3 \mathrm{H}) ;{ }^{13} \mathrm{C}$ NMR $(100 \mathrm{MHz}$, DMSO- $\left.d_{6}\right) \delta: 172.3,172.2,172.0,171.9,171.7,144.4$, 124.1, 79.7, 71.2, 69.9, 68.3, 67.1, 64.7, 59.9, 59.6, 57.4, $52.5,49.7,31.8,31.3,30.3,30.9,29.3,29.2,26.9,26.6$, 24.7, 22.6, 14.5; HRMS (MALDI-TOF) calcd for $\mathrm{C}_{95} \mathrm{H}_{155} \mathrm{~N}_{14} \mathrm{O}_{29}$ 1956.1056, found1956.1079.

\section{2 .9 化合物 FA-DNJ-C6 的合成}

将化合物 FA-AcDNJ-C6 (110 mg, $0.056 \mathrm{mmol})$, 甲 醇钠 $(24 \mathrm{mg}, 0.45 \mathrm{mmol})$ 和 $10 \mathrm{~mL}$ 无水甲醇在室温下摚 拌 $5 \mathrm{~h}$. 旋干溶剂, 剩余物用 $5 \mathrm{~mL}$ 水溶解, 透析袋(保留 分子量 1000)透析 $10 \mathrm{~h}$, 冷冻干燥得浅黄色固体 $75 \mathrm{mg}$, 产率 92\%. m.p. $118.3 \sim 124.4{ }^{\circ} \mathrm{C} ;{ }^{1} \mathrm{H}$ NMR $(600 \mathrm{MHz}$, DMSO- $\left.d_{6}\right) \delta: 8.01(\mathrm{~s}, 3 \mathrm{H}$, Triazole-H), $4.45(\mathrm{~s}, 6 \mathrm{H}), 4.31$ (t, $J=7.2 \mathrm{~Hz}, 6 \mathrm{H}), 3.69$ (d, $J=10.8 \mathrm{~Hz}, 3 \mathrm{H}), 3.60$ (s, 6H), $3.21 \sim 3.15(\mathrm{~m}, 3 \mathrm{H}), 3.04(\mathrm{t}, J=9.0 \mathrm{~Hz}, 3 \mathrm{H}), 2.98(\mathrm{t}, J=$ $7.2 \mathrm{~Hz}, 2 \mathrm{H}), 2.91$ (t, $J=8.4 \mathrm{~Hz}, 3 \mathrm{H}), 2.78 \sim 2.76(\mathrm{~m}, 3 \mathrm{H})$, $2.78 \sim 2.76(\mathrm{~m}, 3 \mathrm{H}), 2.71 \sim 2.66(\mathrm{~m}, 3 \mathrm{H}), 2.36 \sim 2.31(\mathrm{~m}$, $3 \mathrm{H}), 2.30 \sim 2.27(\mathrm{~m}, 2 \mathrm{H}), 2.23 \sim 2.20(\mathrm{~m}, 2 \mathrm{H}), 1.93 \sim 1.88$ (m, $6 \mathrm{H}), 1.79 \sim 1.77(\mathrm{~m}, 6 \mathrm{H}), 1.33 \sim 1.36(\mathrm{~m}, 8 \mathrm{H}), 1.21 \sim$ $1.23(\mathrm{~m}, 44 \mathrm{H}), 0.85(\mathrm{t}, J=7.2 \mathrm{~Hz}, 3 \mathrm{H}) ;{ }^{13} \mathrm{C}$ NMR $(150$ MHz, DMSO- $\left.d_{6}\right) \delta: 171.71,171.24,143.92,123.50,78.99$, $70.64,69.26,67.94,64.16,58.96,56.79,51.95,49.26$, $47.89,31.34,31.23,30.74,29.70,29.02,28.95,28.72$, 26.35 (C-16), 25.81, 24.20, 22.03, 13.89; HRMS (MAL- 
DI-TOF) calcd for $\mathrm{C}_{71} \mathrm{H}_{131} \mathrm{~N}_{14} \mathrm{O}_{17}$ 1451.9815, found 1451.9811

\section{3 表面张力实验}

FA-DNJ-C6 配置成浓度为 $4 \times 10^{-6} \sim 5 \times 10^{-4} \mathrm{~mol} / \mathrm{L}$ 的溶液, $\mathrm{pH}$ 值为 7.0 (PBS, $20 \mathrm{mmol} / \mathrm{L})$, 移液器吸取 200 $\mu \mathrm{L}$ 测试溶液, 安装固定, 悬滴法测定, 用 Laplace-Young 公式计算出表面张力 $\gamma, 25{ }^{\circ} \mathrm{C}$ 下每个样品测五次求平均 值.

\section{4 动态光散射实验}

将 FA-DNJ-C6 溶解在柠檬酸-磷酸盐缓冲溶液中, 浓度为 $1 \times 10^{-4} \mathrm{~mol} / \mathrm{L}$, 用盐酸/氢氧化钠溶液将 $\mathrm{pH}$ 值分 别调至 $2.0,3.0,4.0,5.0,6.0$ 和 7.0. 在 $80{ }^{\circ} \mathrm{C}$ 水浴下加热 $10 \mathrm{~min}$, 超声作用 $30 \mathrm{~min}$, 室温静置 $10 \mathrm{~h}$, 过 $0.45 \mu \mathrm{m}$ 滤 膜后测试溶液粒经分布.

\section{5 透射电子显微镜(TEM)}

将 $10 \mu \mathrm{L} 1 \times 10^{-4} \mathrm{~mol} / \mathrm{L}$ 的 FA-DNJ-C6 溶液滴到 400 目铜网, 静置 $1 \mathrm{~min}$, 滤纸吸走多余溶液, 铜网放入 真空干燥器中干燥 $24 \mathrm{~h}$.

\section{6 糖苷酶抑制活性实验}

将对硝基苯酚(PNP)溶于 $5 \mathrm{~mL}$ 柠檬酸-磷酸盐缓冲 液 $(\mathrm{pH}=5.5,100 \mathrm{mmol} / \mathrm{L})$ 或 $\mathrm{PBS}$ 缓冲液 $(\mathrm{pH}=6.8$ 或 7.3 , $0.1 \mathrm{mmol}$ )中, 最终浓度为 $20 \mathrm{mmol} / \mathrm{L}$, 经多次稀释配制 一系列浓度的溶液. 将上述溶液置于 96 孔微量测定培 养板中, 加入 $\mathrm{Na}_{2} \mathrm{CO}_{3}$ 溶液, 然后用酶标仪在 $405 \mathrm{~nm}$ 波 长下测量每个孔的吸光度, 以浓度对吸光值作图.

分光光度法测定了化合物的酶抑制活性, 实验分为 空白组、对照组、实验空白组和实验组. 在 96 孔板中依 次加入缓冲液、化合物溶液和酶溶液, 每组 3 个平行实 验, 混合均匀 $3 \mathrm{~min}$, 于 $37{ }^{\circ} \mathrm{C}$ 培养箱中保温 $10 \mathrm{~min}$. 底 物在酶作用下能水解产生相应的糖和 PNP, PNP 在 405 $\mathrm{nm}$ 处有最大吸收, 测定其吸光度. 通过反应速率对底 物浓度的双倒数曲线, 得到浓度不同的抑制剂直线斜 率, 以斜率与抑制剂的浓度作图, 得到酶抑制活性 $K_{\mathrm{i}}$ 值.

\section{辅助材料 (Supporting Information) 化合物 7、}

FA-AcDNJ-C6 和 FA-DNJ-C6 的 ${ }^{1} \mathrm{H}$ NMR、 ${ }^{13} \mathrm{C}$ NMR 和 HRMS 谱图, 阳性对照化合物(DNJ-1)糖苷酶抑制活性 的 Lineweaver-Burk 曲线图等. 这些材料可以免费从本 刊网站(http://sioc-journal.cn/)上下载.

\section{References}

[1] Lahin, R.; Ansan, A. A.; Vankar, Y. D. Chem. Soc. Rev. 2013, 42, 5102.

[2] Van de Laar, F. A. P.; Lucassen, L.; Akkermans, R. P.; Van de Lisdonk, E. H.; Rutten, G. E.; Van Weel, C. Cochrane Database Syst.
Rev. 2005, 18, CD003639.

[3] Kato, A.; Nakagome, I.; Sato, K.; Yamamoto, A.; Adachi, I. R.; Nash, J.; Fleet, G. W.; Natori, Y.; Watanabe, Y.; Imahori, T.; Yoshimura, Y.; Takahata, H.; Hirono, S. Org. Biomol. Chem. 2016, 14, 1039.

[4] (a) Pili, R.; Chang, J.; Partis, R. A.; Mueller, R. A.; Chrest, F. J.; Passaniti, A. Cancer Res. 1995, 55, 2920.

(b) Wang, R. J.; Yang, C. H.; Hu, M. L. J. Agric. Food Chem. 2010 , 58,8988 .

[5] (a) Illescas, B. M.; Rojo, J.; Delgado, R.; Martín, N. J. Am. Chem. Soc. 2017, 139, 6018.

(b) Chavan, S. R.; Gavale, K. S.; Khan, A.; Joshi, R.; Kumbhar, N.; Chakravarty, D.; Dhavale, D. D. ACS Omega 2017, 2, 7203.

(c) Kiappes, J. L.; Hill, M. L.; Alonzi, D. S.; Miller, J. L.; Iwaki, R.; Sayce, A. C.; Caputo, A. T.; Kato A.; Zitzmann, N. ACS Chem. Biol. 2018, 13, 60 .

[6] (a) Choukhi, A.; Ung, S.; Wychowski, C.; Dubuisson, J. J. Virol. 1998, 72, 3851.

(b) Babu, Y. S.; Chand, P.; Kotian, P. L. Annu. Rep. Med. Chem. 2006, 41, 287.

[7] (a) Howard, E.; Cousido-Siah, A.; Lepage, M. L.; Schneider, J. P.; Bodlenner, A.; Mitschler, A.; Meli, A.; Izzo, I.; Alvarez, A.; Podjarny, A.; Compain, P. Angew. Chem., Int. Ed. 2018, 57, 8002. (b) Compain, P.; Bodlenner, A. ChemBioChem 2014, 15, 1239.

(c) Kanfar, N.; Bartolami, E.; Zelli, R.; Marra, A.; Winum, J. Y.; Ulrich, S.; Dumy, P. Org. Biomol. Chem. 2015, 13, 9894.

(d) Ortiz Mellet, C.; Nierengarten, J. F.; García Fernández, J. M. J. Mater. Chem. B 2017, 5, 6428 .

(e) Zhang, H. C.; Hao, A. Y.; Du, G. Y.; Shen, J. Chin. J. Org Chem. 2008, 28, 1515 (in Chinese). (张华承，郝爱友，杜光焰，申健，有机化学, 2008, 28, 1515.)

[8] (a) Rísquez-Cuadro, R.; Fernández, J. M. G.; Nierengarten, J. F.; Mellet, C. O. Chem.-Eur. J. 2013, 19, 16791.

(b) Joosten, A.; Schneider, J. P.; Lepage, M. L.; Tarnus, C.; Bodlenner A.; Compain, P. Eur. J. Org. Chem. 2014, 1866.

(c) Decroocq, C.; Rodríguez-Lucena, D.; Russo, V.; Barragán, T. M.; Mellet, C. O.; Compain, P. Chem.-Eur. J. 2011, 17, 13825.

(d) Alvarez-Dorta, D.; King, D. T.; Legigan, T.; Ide, D.; Adachi, I.; Deniaud, D.; Désiré, J.; Kato, A.; Vocadlo, D.; Gouin, S. G.; Blériot, Y. Chem.-Eur. J. 2017, 23, 9022

(e) Brissonnet, Y.; Ladevèze, S.; Tezé, D.; Fabre, E.; Deniaud, D.; Daligault, F.; Tellier, C.; Sěsták, S.; Remaud-Simeon, M.; Potocki-Veronese G.; Gouin, S. G. Bioconjugate Chem. 2015, 26, 766.

(f) Lepage, M. L.; Schneider, J. P.; Bodlenner, A.; Meli, A.; Schmitt, F. D.; Tarnus, M.; Nguyen-Huynh, C.; Francois, N. T.; Riccardis, Y. N.; Lize-Wagner, E. L.; Birck, C.; Cousido-Siah, A.; Podjarny, A.; Izzo, I.; Compain, P. Chem.-Eur. J. 2016, 22, 5151.

[9] Decroocq, C.; Joosten, A.; Sergent, R.; Barragán, T. M.; Mellet, C. O.; Compain, P. ChemBioChem 2013, 14, 2038.

[10] Nierengarten, J. F.; Schneider, J. P.; Trinh, T. M. N.; Joosten, A.; Holler, M.; Lepage, M. L.; Bodlenner, A.; García-Moreno, M. I.; Mellet, C. O.; Compain, P. Chem.-Eur. J. 2018, 24, 2483.

[11] (a) McClements, D. J. Food Funct. 2018, 9, 22.

(b) Wu, D.; Shen, J.; Bai, H. Z.; Yu, G. C. Chem. Commun. 2018 , 54, 2922.

(c) Yeh, H. W.; Lin, T. S.; Wang, H. W.; Cheng, H. W.; Liu, D. Z.; Liang, P. H. Org. Biomol.Chem. 2015, 13, 11518.

[12] (a) Barnard, A.; Smith, D. K. Angew. Chem., Int. Ed. 2012, 51, 6572 .

(b) Chen,Y.; Liu, Y. J. Chin. J. Org. Chem. 2012, 32, 805 (in Chinese).

(陈湧, 刘育, 有机化学, 2012, 32,805.)

[13] Yamabe, M.; Kaihatsu, K.; Ebara, Y. Bioconjugate Chem. 2018, 29 , 1490.

[14] (a) Wang, K. R.; An, H. W.; Wang, Y. Q.; Zhang, J. C.; Li, X. L. Org. Biomol. Chem. 2013, 11, 1007.

(b) Yeung, S. Y.; Sergeeva, Y.; Dam, T.; Jönsson, P.; Pan, G. Q. 
Chaturvedi, V.; Sellergren, B. Langmuir 2019, 35, 8174.

[15] Qi, Z. H.; Bharate, P.; Lai, C. H.; Ziem, B.; Böttcher, C.; Schulz, A.; Beckert, F.; Hatting, B.; Mülhaupt, R.; Seeberger, P. H.; Haag, R. Nano Lett. 2015, 15, 6051.

[16] Bonduelle, C.; Huang, J.; Mena-Barragán, T.; Mellet, C. O.; Decroocq, C.; Etamé, E.; Heise, A.; Compain, P.; Lecommandoux, S. Chem. Commun. 2014, 50, 3350.

[17] Li, J. J.; Wang, K. R.; Li, R. F.; Yang, J. X.; Li, M.; Zhang, H. X.; Cao, Z. R.; Li, X. L. J. Mater. Chem. B 2019, 7, 1270.

[18] Li, M.; Wang, K. R.; Yang, J. X.; Peng, Y. T.; Liu, Y. X.; Zhang, H. X.; Li, X. L. J. Mater. Chem. B 2019, 7, 1379.

[19] Decroocq, C.; Rodríguez-Lucena, D.; Ikeda, K.; Asano, N.; Compain, P. ChemBioChem 2012, 13, 661.

[20] Ma, C.-L. M.S. Thesis, Hebei University, Baoding, 2016 (in Chinese)

(马翠兰, 硕士论文, 河北大学, 保定, 2016.)

[21] Kang, Y.; Ha, W.; Liu, Y. Q.; Ma, Y.; Fan, M. M.; Ding, L. S.;
Zhang, S.; Li, B. J. Nanotechnology 2014, 25, 335101.

[22] Roy, S.; Dey, J. Langmuir 2005, 21, 10362.

[23] (a) Trinh, T. M. N.; Holler, M.; Schneider, J. P.; García-Moreno, M. I.; Fernández, J. M. G.; Bodlenner, A.; Compain, P.; Mellet, C. O.; Nierengarten, J. F. J. Mater. Chem. B 2017, 5, 6546.

(b) Lepage, M. L.; Schneider, J. P.; Bodlenner, A.; Meli, A. Riccardis, F. D.; Schmitt, M.; Tarnus, C.; Nguyen-Huynh, N.-T.; Francois, Y. N.; Leize-Wagner, E.; Birck, C.; Cousido-Siah, A.; Podjarny, A.; Izzo, I.; Compain, P. Chem.-Eur. J. 2016, 22, 5151.

[24] Wang, K. R.; Guo, D. S.; Jiang, B. P.; Sun, Z. H.; Liu, Y. J. Phys. Chem. B 2010, 114, 101.

[25] Diot, J.; García-Moreno, M. I.; Gouin, S. G.; Mellet, C. O.; Haupt, H.; Kovensky, J. Org. Biomol. Chem. 2009, 7, 357.

[26] Howar, E.; Cousido-Siah, A.; Lepage, M. L.; Schneider, J. P.; Bodlenner, A.; Mitschler, A.; Meli, A.; Izzo, I.; Alvarez, H. A.; Podjarny, A.; Compain, P. Angew. Chem., Int. Ed. 2018, 57, 8002. 